\title{
EFFECT OF TEMPERATURE ON THE CAVITATION EROSION OF CAST IRON
}

\author{
YOSHIRO IWAI* and TSUNENORI OKADA \\ Department of Mechanical Engineering, Fukui University, Bunkyo 3-9-1, Fukui (Japan) \\ F. G. HAMMITT
}

Department of Mechanical Engineering, The University of Michigan, Ann Arbor, MI 48109 (U.S.A.)

(Received May 14, 1982; in revised form August 2, 1982)

\section{Summary}

Vibratory cavitation erosion tests of gray cast iron, together with tests of tool steel and 316 stainless steel for comparison, were performed at various water temperatures and horn amplitudes under a suppression pressure of 1 bar. The erosion processes for cast iron under the highest temperatures used $\left(200\right.$ and $230{ }^{\circ} \mathrm{F}$, i.e. 93 and $\left.110^{\circ} \mathrm{C}\right)$ are similar to those at room temperature. For each of the materials tested, the maximum weight loss rate increases, shows a peak and then decreases with increasing temperature. However, the maximum damage temperature for cast iron decreases with amplitude, i.e. 200,170 and $160^{\circ} \mathrm{F}\left(93,77\right.$ and $\left.71^{\circ} \mathrm{C}\right)$ for double-horn amplitudes of $1.0 \times 10^{-3}, 1.38 \times 10^{-3}$ and $1.78 \times 10^{-3}$ in $(25.4,35.1$ and $45.2 \mu \mathrm{m}$ ). The peak for tool steel and 316 stainless steel occurs at $160^{\circ} \mathrm{F}$ $\left(71^{\circ} \mathrm{C}\right)$ regardless of amplitude. Liquid temperature effects for cast iron erosion were explained by considering the interrelation between corrosive action and mechanical action due to cavitation bubble collapse.

\section{Introduction}

Cast iron is used extensively for components exposed to cavitation erosion in hydraulic machinery, internal combustion engines and other devices. These components are often used in high temperature liquids, so that the effect of temperature on cavitation erosion for cast iron becomes a significant problem.

Temperature effects on cavitation erosion have been studied in water, liquid metals and other fluids by a number of investigators (e.g. in refs. 1 7). Most of these papers indicate that the maximum damage rate occurs at a

*Visiting Assistant Professor, Cavitation and Multiphase Flow Laboratory, Department of Mechanical Engineering, The University of Michigan, Ann Arbor, MI 48109, U.S.A., from 1981 to 1982 . 
temperature roughly midway between the freezing and boiling points for the liquid at the test pressure. This subject has been investigated using various materials such as aluminum alloys, carbon steel, stainless steel and cast iron (e.g. in refs. 8 and 9). A maximum damage rate $\mathrm{MDPR}_{\max }$ exists at water temperatures of about $140-160^{\circ} \mathrm{F}\left(60-71{ }^{\circ} \mathrm{C}\right)$, but the differences in the temperature effects on erosion rates among different materials were not made clear.

Plesset [4] reported that the maximum erosion temperature increases for mild steel (a corrosion-sensitive material) when the test liquid is changed from distilled water to $3 \%$ salt solution. Thus the corrosion sensitivity of the material is a significant factor. The physical conditions of the liquid, such as its vapor pressure, are also important.

Cast iron is a brittle material because of its graphite component and thus generates locally deep erosion pits on the surface in cavitation erosion [10]. It thus seems probable that cast iron erosion is strongly affected by the liquid properties and accordingly may exhibit temperature effects on erosion different from other materials.

In this work, vibratory cavitation erosion tests of gray cast iron, together with tests of a tool steel and 316 stainless steel for comparison, were carried out at various water temperatures and horn amplitudes at a suppression pressure of 1 bar. The effects of temperature on cast iron erosion are discussed considering the interrelations between corrosive action and mechanical action due to cavitation bubble collapse.

\section{Test material and experimental procedure}

\subsection{Specimen material}

The test material was a gray cast iron of tensile strength $20.4 \mathrm{klbf} \mathrm{in}^{-2}$ $\left(14.4 \mathrm{kgf} \mathrm{mm}^{-2}\right)$. For comparison, a tool steel and 316 stainless steel (18\% $\mathrm{Cr}, 13 \% \mathrm{Ni}$ ) were also used. The cast iron and 316 stainless steel were prepared at the University of Michigan and the tool steel at Fukui University. The chemical compositions and mechanical properties are listed in Tables 1 and 2. The properties of the 316 stainless steel were measured for an ASTM Committee G-2 cavitation round robin [11]. The Brinell hardness $H_{\mathrm{B}}$ gives the macrohardness and the Vickers hardness $H_{\mathrm{V}}$ the microhardness of the material.

\section{TABLE 1}

Chemical compositions (\%) of the materials

\begin{tabular}{llllllll}
\hline Material & $C$ & Si & $M n$ & $P$ & $S$ & $C r$ & $N i$ \\
\hline Cast iron & 3.20 & 1.57 & 0.50 & 0.025 & 0.057 & - & - \\
Tool steel & 1.11 & 0.34 & 0.98 & 0.014 & 0.009 & - & - \\
$\begin{array}{l}316 \\
\text { stainless steel }\end{array}$ & 0.06 & 0.50 & 1.50 & - & - & 18.00 & 13.00 \\
\hline
\end{tabular}


TABLE 2

Mechanical properties of the materials at room temperature

\begin{tabular}{lcll}
\hline Material & $\begin{array}{l}\sigma_{\mathrm{B}} \\
\left(\mathrm{klbf} \mathrm{in}^{-2}\left(\mathrm{kgf} \mathrm{mm}^{-2}\right)\right)\end{array}$ & $\begin{array}{l}H_{\mathrm{B}} \\
(\mathrm{HB})\end{array}$ & $\begin{array}{l}H_{\mathrm{V}} \\
(\mathrm{HV})\end{array}$ \\
\hline $\begin{array}{l}\text { Cast iron } \\
\text { Tool steel }\end{array}$ & $20.4(14.4)$ & 184 & 234 \\
$\begin{array}{l}316 \\
\text { stainless steel }\end{array}$ & $161.7(113.9)$ & 331 & 370 \\
\hline
\end{tabular}

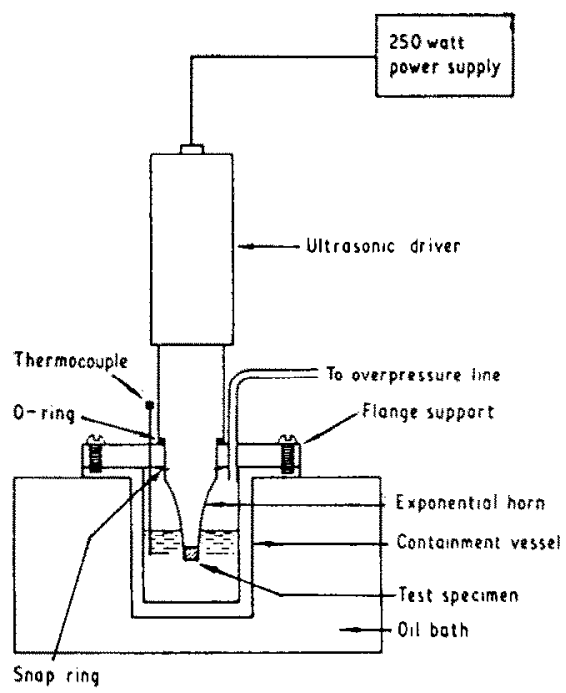

Fig. 1. Schematic diagram of the vibratory cavitation facility.

\subsection{Experimental procedure}

Cavitation erosion tests were carried out using the University of Michigan piezoelectric vibratory horn facility (Fig. 1). The horn frequency was 20 $\mathrm{kHz}$ and peak-to-peak amplitudes were $1.0 \times 10^{-3}, 1.38 \times 10^{-3}$ and $1.78 \times$ $10^{-3}$ in $(25.4,35.1$ and $45.2 \mu \mathrm{m})$. The stainless steel test vessel allows a hydrostatic pressure to be applied to the liquid and the temperature to be controlled. Four temperatures $\left(80,160,200\right.$ and $230^{\circ} \mathrm{F}(27,71,93$ and $110^{\circ} \mathrm{C}$ )) were used. The suppression pressure $p_{\mathrm{sv}}=p-p_{\mathrm{v}}$ (where $p$ is the static pressure and $p_{\mathrm{v}}$ is the vapor pressure), which was supplied by air, was kept at 1 bar under different temperatures. Fresh tap water (Ann Arbor) was used as the test liquid. No measurements of gas content were made.

The specimen was a cylinder $9 / 16$ in $(14.3 \mathrm{~mm})$ in diameter. Specimen surfaces were polished with 600 grade emery paper. The weight loss was measured with a precision balance (sensitivity, $0.01 \mathrm{mgf}$ ). 


\section{Experimental results}

\subsection{Cavitation erosion of cast iron}

Figures 2 - 5 show curves of the weight loss rate, i.e. the weight loss per unit test duration, versus test duration for various amplitudes $\left(1.0 \times 10^{-3}\right.$, $1.38 \times 10^{-3}$ and $1.78 \times 10^{-3}$ in) for water temperatures of $80^{\circ} \mathrm{F}, 160^{\circ} \mathrm{F}$, $200{ }^{\circ} \mathrm{F}$ and $230^{\circ} \mathrm{F}$ respectively. Generally, the erosion process of cast iron
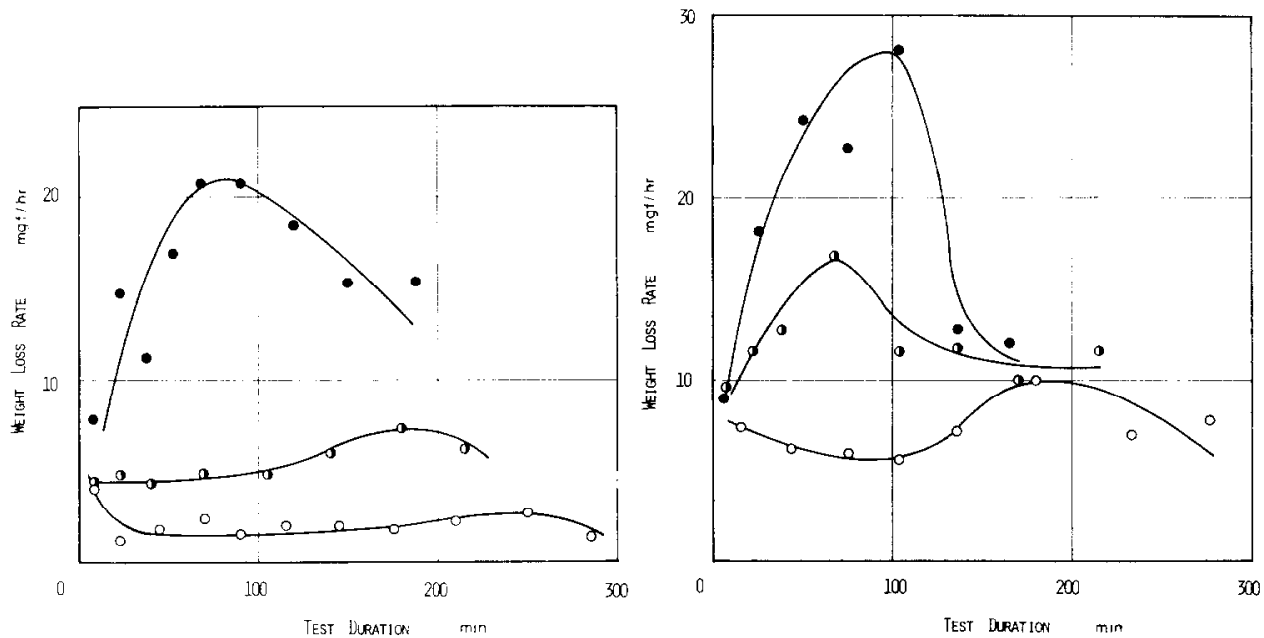

Fig. 2. Weight loss rate us. test duration for a water temperature of $80^{\circ} \mathrm{F}: 0,1.0 \times 10^{-3}$ in; $1.38 \times 10^{-3}$ in;, $1.78 \times 10^{-3}$ in.

Fig. 3. Weight loss rate us. test duration for a water temperature of $160^{\circ} \mathrm{F}: 0,1.0 \times 10^{-3}$ in;, $1.38 \times 10^{-3}$ in; $\bullet 1.78 \times 10^{-3}$ in.
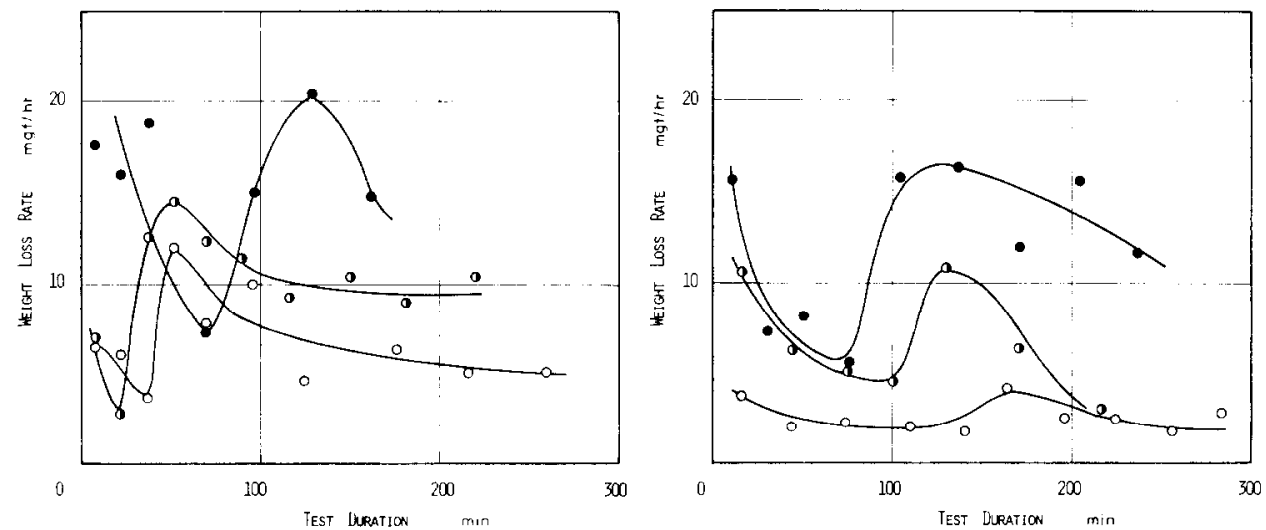

Fig. 4. Weight loss rate $v$ s. test duration for a water temperature of $200^{\circ} \mathrm{F}: 0,1.0 \times 10^{-3}$ in; $\bullet, 1.38 \times 10^{-3}$ in; $\bullet, 1.78 \times 10^{-3}$ in.

Fig. 5. Weight loss rate us. test duration for a water temperature of $230^{\circ} \mathrm{F}: 0,1.0 \times 10^{-3}$ in; $\bullet, 1.38 \times 10^{-3}$ in; $\bullet 1.78 \times 10^{-3}$ in. 
can be divided into several stages, an initial period, an incubation period, a transition period and a stable period, which are similar to those found for carbon steel $[10]$.

In the present tests the weight loss rate for all temperatures increases to a maximum after the initial and incubation periods except for a few cases in which it then decreases gradually with test duration. The maximum weight loss rate increases with increasing amplitude, although the precise point of maximum rate is less evident for low amplitudes. For high amplitudes at 80 and $160^{\circ} \mathrm{F}$ the weight loss rate attains a maximum without a discernible incubation period because the initial and incubation periods are completed within the first test interval as a consequence of the greater damage rate. However, it was found that cavitation erosion of cast iron proceeds in a similar fashion even when the water temperature is increased from room temperature to the highest value $\left(230^{\circ} \mathrm{F}\right)$. These erosion stages are close to those suggested by Thiruvengadam and Preiser [12]. We discuss here the temperature effect especially for the maximum weight loss rate $W L R_{\max }$.

Figure 6 shows the relation between maximum weight loss rate $W L R_{\max }$ and amplitude on a log-log plot. A linear relation is obtained for every temperature, although the line slopes are different. Thus the expected relation $\mathrm{WLR}_{\max }=k$ (amplitude $)^{n}$, where $k$ depends on the material tested, is obtained. The values of the exponent $n$ are listed in Table 3 for various water temperatures. Thiruvengadam and Preiser [12] found that the cavitation damage rate in distilled water $\left(80^{\circ} \mathrm{F}\right)$ was proportional to the square of

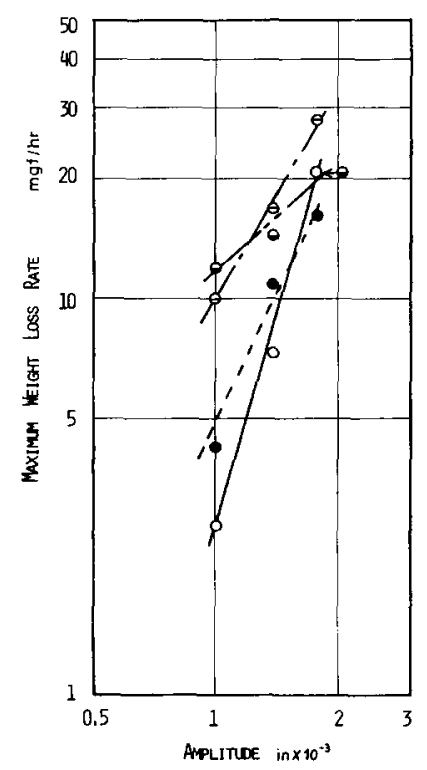

Fig. 6. Relation between maximum weight loss rate and amplitude for cast iron: $0,80^{\circ} \mathrm{F}$; $\ominus, 160^{\circ} \mathrm{F} ; \odot, 200^{\circ} \mathrm{F} ; \bullet, 230^{\circ} \mathrm{F}$. 
TABLE 3

The values of the damage exponent $n$ for cast iron, tool steel and 316 stain less steel for various water temperatures

\begin{tabular}{lllll}
\hline Material & \multicolumn{4}{l}{ Values of $n$ for the following lemperalures } \\
\cline { 2 - 5 } & $80^{\circ} \mathrm{F}$ & $160^{\circ} \mathrm{F}$ & $200^{\circ} \mathrm{F}$ & $230^{\circ} \mathrm{F}$ \\
\hline Cast iron & 3.5 & 1.8 & 0.9 & 2.4 \\
Tool steel & 3.3 & 2.2 & 2.5 & - \\
$\begin{array}{l}316 \text { stainless } \\
\text { steel }\end{array}$ & 3.8 & 3.9 & 4.1 & - \\
\hline
\end{tabular}

the horn amplitude. Similar relationships, but with different exponents, have been found (e.g. in refs. 6 and 8 ). By contrast, an exponent $n=3.5$ was obtained at room temperature in the present experiment, i.e. $n$ was larger than expected. This may be due to the fact that the exponent depends on test parameters such as the frequency of the oscillator and the specimen diameter. However, the discrepancy cannot be fully explained.

On the basis of data from Fig. 6, the variations in maximum weight loss rate (for constant amplitude) versus temperature are shown in Fig. 7. For any amplitude the maximum weight loss rate first increases, then passes through a peak and finally decreases, with increasing temperature. The peak damage temperature decreases with amplitude, i.e. 200,170 and $160^{\circ} \mathrm{F}$ for amplitudes of $1.0 \times 10^{-3}, 1.38 \times 10^{-3}$ and $1.78 \times 10^{-3} \mathrm{in}$. The temperature effect on cast iron thus depends on the cavitation intensity. These results have not been reported before since most previous investigations were performed at constant amplitude.

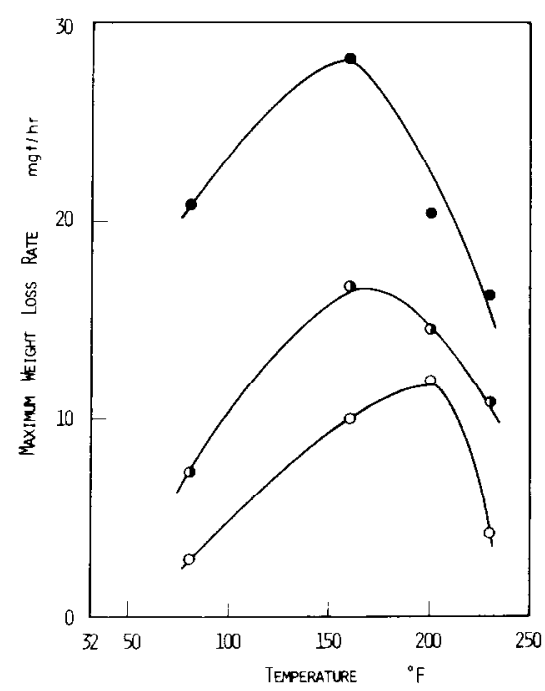

Fig. 7. Relation between maximum weight loss rate and temperature for cast iron: 0 , $1.0 \times 10^{-3} \mathrm{in} ; \boldsymbol{\bullet}, 1.38 \times 10^{-3} \mathrm{in} ; \bullet, 1.78 \times 10^{-3} \mathrm{in}$. 

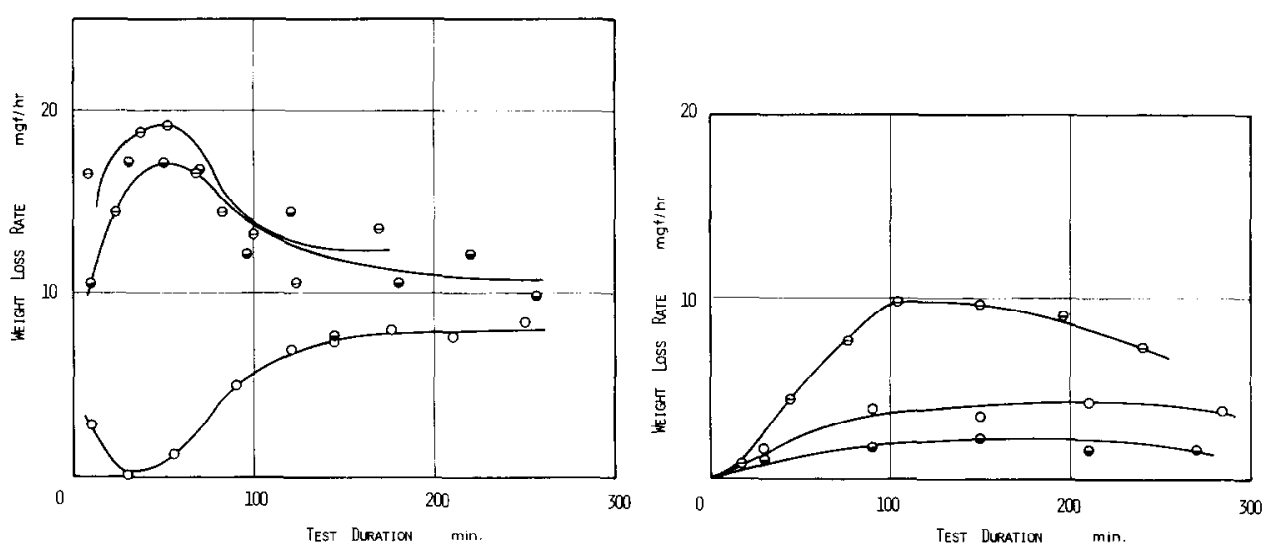

Fig. 8. Weight loss rate vs. test duration for tool steel (amplitude, $1.78 \times 10^{-3} \mathrm{in}$ ): 0 , $80^{\circ} \mathrm{F} ; \ominus, 160^{\circ} \mathrm{F} ; \ominus, 200^{\circ} \mathrm{F}$.

Fig. 9. Weight loss rate vs. test duration for 316 stainless steel (amplitude, $1.78 \times 10^{-3} \mathrm{in}$ ): $\odot, 80^{\circ} \mathrm{F} ; \ominus, 160^{\circ} \mathrm{F} ; \ominus, 200^{\circ} \mathrm{F}$.

\subsection{Cavitation erosion of tool steel and 316 stainless steel}

In order to compare cast iron with other metals, cavitation erosion tests were also carried out using high carbon $(1.1 \% \mathrm{C})$ tool steel and 316 stainless steel, a relatively non-corrosive material. The weight loss rates for the tool steel and 316 stainless steel, obtained at various temperatures and at an amplitude of $1.78 \times 10^{-3}$ in, are shown in Figs. 8 and 9. The erosion stages for both of these materials are similar to those for cast iron.

Figures 10 and 11 show the relation between the maximum weight loss rate and the amplitude for the tool steel and 316 stainless steel. Reliable results for 316 stainless steel at an amplitude of $1.0 \times 10^{-3}$ in could not be obtained because of the small weight loss rate. $W L R_{\text {max }}$ exponents are calculated and listed in Table 3.

\section{Discussion}

To consider the effects of liquid temperature on the cavitation erosion of cast iron, the relations between maximum weight loss rate and temperature for tool steel and 316 stainless steel are shown in Fig. 12 together with those obtained for cast iron (from Fig. 7). For tool steel and 316 stainless steel a peak of maximum weight loss rate occurs at $160^{\circ} \mathrm{F}$ regardless of amplitude. Similar results were obtained previously for AISI-SAE 1018 carbon steel $[8,9]$.

The temperature-effect mechanisms for the 316 stainless steel, which involve little or no corrosive effect, are considered to be as follows and are also explained elsewhere [1 - 5]. The quantity of dissolved air decreases and the vapor pressure increases slightly as the water temperature increases from 

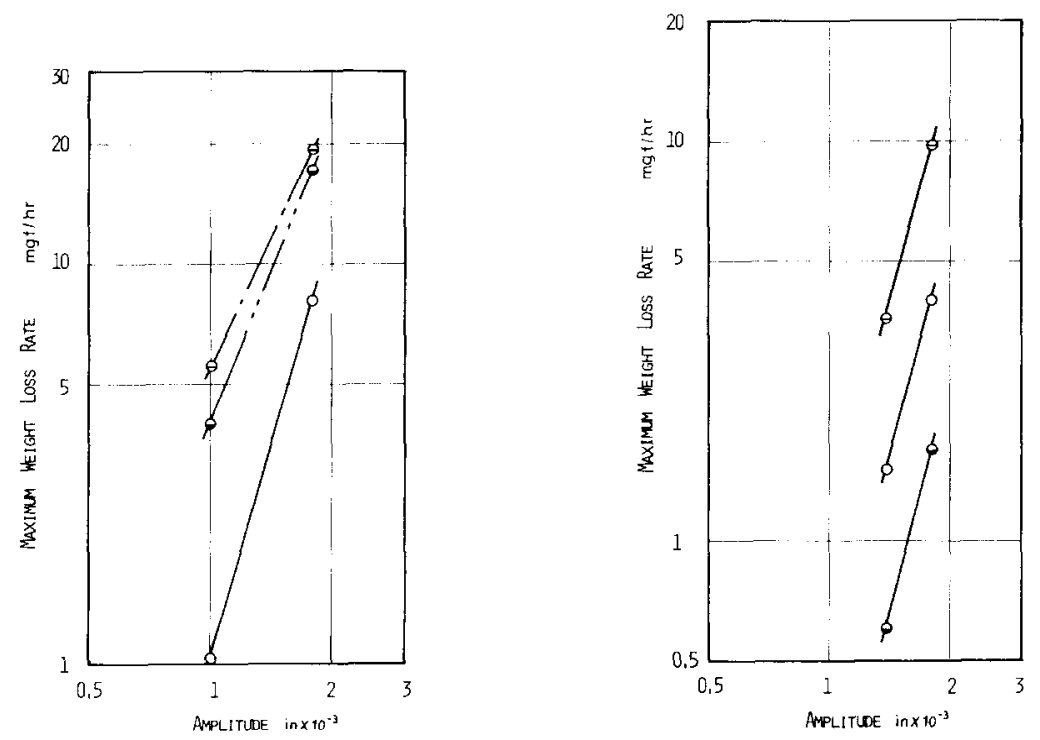

Fig. 10. Relation between maximum weight loss rate and amplitude for tool steel: 0 , $80^{\circ} \mathrm{F} ; \ominus, 160^{\circ} \mathrm{F} ; \ominus, 200^{\circ} \mathrm{F}$.

Fig. 11. Relation between maximum weight loss rate and amplitude for 316 stainless steel: $0,80^{\circ} \mathrm{F} ; \ominus, 160^{\circ} \mathrm{F} ; \ominus, 200^{\circ} \mathrm{F}$.
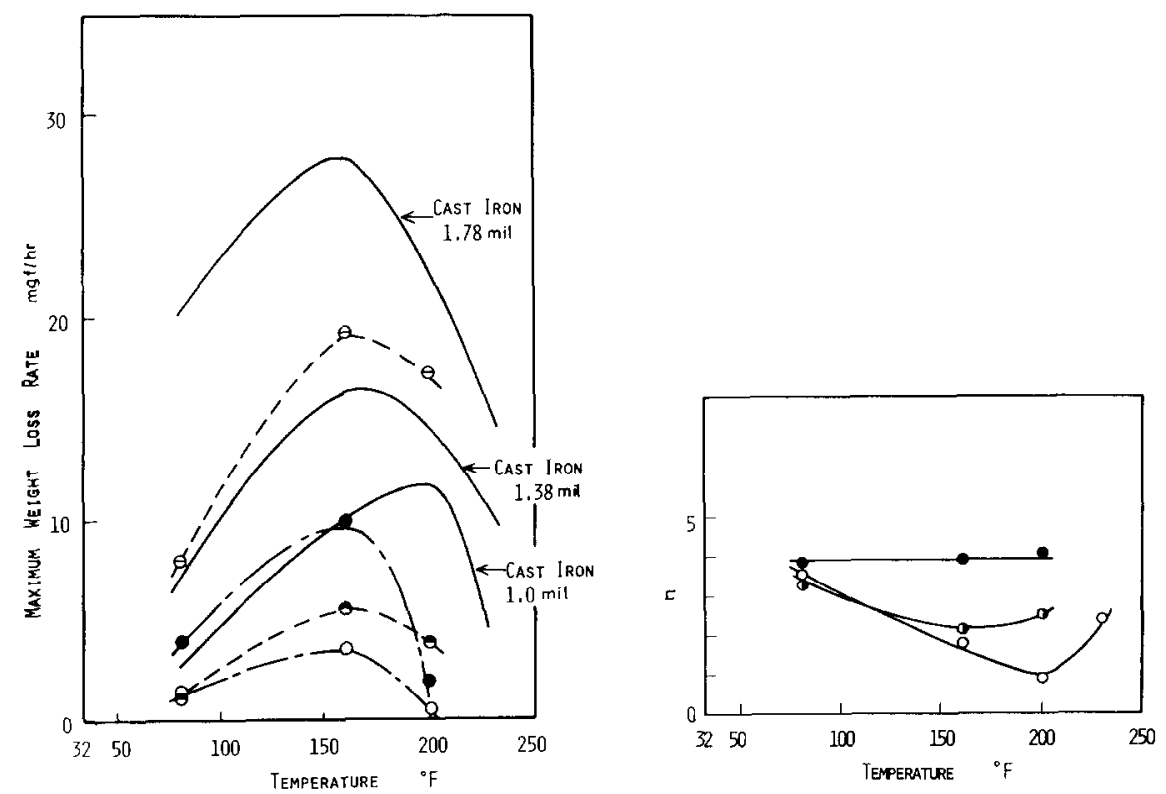

Fig. 12. Relation between maximum weight loss rate and temperature for various materials: $O, 316$ stainless steel, $1.38 \times 10^{-3}$ in; $\bullet, 316$ stainless steel, $1.78 \times 10^{-3}$ in; $\bullet$, tool steel, $1 \times 10^{-3}$ in; $\theta$, tool steel, $1.78 \times 10^{-3}$ in.

Fig. 13. Damage exponent $n$ us. temperature for various materials: $\odot$, cast iron; $\odot$, tool steel; $\bullet, 316$ stainless steel. 
room temperature to a relatively low temperature $\left(120^{\circ} \mathrm{F}\right)$. Consequently, the cushioning effects on bubble collapse are reduced, whereas the number of bubbles increases, because there is more dissolved air, resulting in an increase in damage because of increased bubble collapse for this low temperature range. Reduction in viscosity and surface tension may also be involved. However, for higher temperatures, the amount of damage decreases strongly because the.cushioning effect (now due to the increased vapor content of the bubbles) increases strongly with the rapid increase in vapor pressure. These variations in the physical conditions of the liquid cause similar behavior for different cavitation intensities. For 316 stainless steel the peak of maximum weight loss rate occurs for the present tests at about $160^{\circ} \mathrm{F}$ for all amplitudes.

The maximum erosion rate of cast iron, however, moves to higher temperatures with decreasing amplitude. This phenomenon is similar to the results for mild steel reported by Plesset [4], i.e. the maximum damage temperature increased when the liquid was changed from distilled water to $3 \%$ salt solution. The temperature effects for cast iron may thus be considered to depend on the variation in chemical action between liquid and material as well as the behavior of collapsing bubbles at higher temperatures. The reduction in mechanical properties for higher temperatures may also affect the cavitation damage. However, it seems to be less important in the shift in maximum damage temperature with amplitude since the variations in mechanical properties can be expected to cause similar behavior for every amplitude.

Under low amplitudes, the eroded surface is easily affected by the liquid environment since particles are actually removed after long cavitation exposure [13]. The degree of chemical action (corrosion rate) for cast iron is known to increase with temperature over the range tested. Thus at $200^{\circ} \mathrm{F}$ erosion cracks propagate more easily because of surface oxidization although the vapor-cushioning effect on bubble collapse is increased. The ejection of erosion particles and the erosion rate are larger for 200 than for $160^{\circ} \mathrm{F}$. However, at $230^{\circ} \mathrm{F}$, although chemical action is reduced by decreased dissolved oxygen, the damage-suppressing "thermodynamic effect" (see for example refs. 1 - 7) is more important so that the erosion rate is greatly reduced compared with $200^{\circ} \mathrm{F}$. For larger horn amplitudes, the surface is fractured by the mechanical action involved in bubble collapse so that the chemical effect almost disappears. Thus the maximum erosion temperature shifts to a lower temperature for a larger horn amplitude. This may also explain the difference in peak damage temperatures found for cast iron at various amplitudes. Tool steel (Fig. 12) has a temperature dependence midway between cast iron and 316 stainless steel.

Values of the damage exponent $n$ are listed in Table 3 for various materials and $n$ is shown in Fig. 13 as a function of temperature. Nearly the same values of $n$ were found for all three materials at $80^{\circ} \mathrm{F}$. However, $n$ decreases with increasing temperature for cast iron and tool steel but increases slightly at $230^{\circ} \mathrm{F}$ for cast iron. For 316 stainless steel $n$ is almost independent of temperature. Corrosive effects on AISI-SAE 1020 mild steel 
greatly alter the effect of amplitude on erosion rate, i.e. $n$ gradually decreases with increasing salt concentration up to $9 \%$. By contrast, there is no effect of liquid corrosivity on $n$ for aluminum alloy 1100-O [14]. Fukui University tests [15] with $3 \%$ salt water show that $n$ is smaller than for ion-exchanged water for the corrosion-sensitive cast iron. Therefore it can be concluded that cavitation damage is strongly affected for cast iron by the corrosive action of tap water at $200^{\circ} \mathrm{F}$ compared with temperatures either below or above that value. Thus the temperature effect on cast iron erosion differs somewhat from that for the other materials tested. This result is due to the combined chemical and mechanical actions of cavitation under higher temperatures.

\section{Conclusions}

Vibratory cavitation erosion tests in tap water on cast iron, tool steel and 316 stainless steel were made with various water temperatures and horn amplitudes. The following conclusions can be drawn.

(1) The erosion processes of cast iron under the highest test temperatures $\left(200\right.$ and $\left.230^{\circ} \mathrm{F}\right)$ are similar to those for room temperature, i.e. the weight loss rate increases up to a maximum value and then decreases gradually with further test duration.

(2) For each material tested, the maximum weight loss rate increases, shows a peak and then decreases with increasing temperature. However, the maximum damage temperature for cast iron decreases with amplitude, i.e. $200^{\circ} \mathrm{F}, 170^{\circ} \mathrm{F}$ and $160^{\circ} \mathrm{F}$ for amplitudes of $1.0 \times 10^{-3} \mathrm{in}, 1.38 \times 10^{-3} \mathrm{in}$ and $1.78 \times 10^{-3}$ in respectively. By contrast, the peak for tool steel and 316 stainless steel occurs at $160^{\circ} \mathrm{F}$ regardless of amplitude.

(3) The relation maximum weight loss rate $=k$ (amplitude) ${ }^{n}$ is confirmed for various temperatures. The values of $n$ are almost the same for cast iron, tool steel and 316 stainless steel at room temperature but cast iron has the smallest value of $n$ at higher temperatures. $n$ depends on temperature for all materials.

(4) Temperature effects for cast iron differ from those obtained for the other materials because of increased chemical action under higher temperatures.

\section{Acknowledgments}

The present study was performed as part of a joint research program between the University of Michigan and Fukui University. Financial support for this work was provided by Office of Naval Research Contract N0001476-C-0697 and National Science Foundation Grant ENG 75-2315 together with internal University of Michigan funds. 


\section{References}

1 F. G. Hammit and D. O. Rogers, Effects of pressure and temperature variation in vibratory cavitation damage test, J. Mech. Eng. Sci., 12 (6) (1970) 432 - 439.

2 R. Garcia and F. G. Hammitt, Cavitation damage and correlations with material and fluid properties, J. Basic Eng., 89 (1967) $753-763$.

3 F. G. Hammitt, Cavitation and Multiphase Flow Phenomena, McGraw-Hill, New York, 1980.

4 M. S. Plesset, Temperature effects in cavitation damage, J. Basic Eng., 94 (1972) 559 566.

5 S. G. Young and J. R. Johnston, Effect of temperature and pressure on cavitation damage in sodium, Characterization and Determination of Erosion Resistance, ASTM Spec. Tech. Publ. 474, 1970, pp. 67 - 108 (ASTM, Philadelphia, PA).

6 J. M. Hobbs, Experience with a 20-kc cavitation erosion test, ASTM Spec. Tech. Publ. 408, 1967, pp. 159 - 185 (ASTM, Philadelphia, PA).

7 W. C. Leith, Prediction of cavitation damage in the alkali liquid metals, Proc., ASTM, 65 (1965) $789-800$.

8 J.-g. He and F. G. Hammitt, Comparison of cavitation erosion test results from Venturi and vibratory facilities, Wear, 76 (1982) 269.292 (also available as DRDA Rep. UMICH 014456-73-I, March 1981 (Division of Research Development and Administration, University of Michigan, Ann Arbor, MI)).

9 Y.-k. Zhou and F. G. Hammitt, Vibratory cavitation test at two bar suppression pressure, DRDA Rep. UMICH 014571-39-I, November 1981 (Division of Research Development and Administration, University of Michigan, Ann Arbor, MI).

$10 \mathrm{~T}$. Okada, Y. Iwai and A. Yamamoto, A study of cavitation erosion of cast iron, DRDA Rep. UMICH 014571-42-I, March 1982 (Division of Research Development and Administration, University of Michigan, Ann Arbor, MI).

11 F. G. Hammitt, C. Chao, C. L. Kling and D. O. Rogers, ASTM round robin tesl with vibratory cavitation and liquid impact facilities of various materials, Mater. Res. Stand., $10(10)(1970) 16-23$.

12 A. Thiruvengadam and $\mathrm{H}$. S. Preiser, On testing materials for cavitation damage resistance, J. Ship Res., 8 (3) (1964) 39 - 56.

13 T. Okada, Corrosive liquid effects on cavitation erosion, J. Ship Res., 25 (4) (1981) $271-284$.

$14 \mathrm{~S}$. Waring, H. S. Preiser and A. Thiruvengadam, On the role of corrosion in cavitation damage, J. Ship Res., 9 (4) (1965) 200 - 208.

15 T. Okada, Y. Iwai and A. Yamamoto, Cavitation erosion of cast iron in $3 \%$ salt water, DRDA Rep. UMICH 014571-43-I, March 1982 (Division of Research Development and Administration, University of Michigan, Ann Arbor, MI). 